\title{
Adjustable and negative thermal expansion from multilayered systems
}

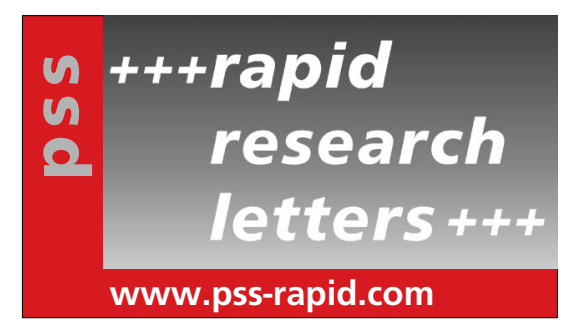

\author{
Joseph N. Grima*, ${ }^{*}$ Ludovica Oliveri', Brian Ellul', Ruben Gatt', Daphne Attard', Gianluca Cicala², \\ and Giuseppe Recca ${ }^{2}$ \\ ${ }^{1}$ Faculty of Science, University of Malta, Msida MSD 2080, Malta \\ ${ }^{2}$ Faculty of Engineering, Department of Physical and Chemical Methodologies for Engineering, University of Catania, \\ Viale A. Doria $n^{\circ} 6,95125$ Catania, Italy
}

Received 10 March 2010, revised 16 April 2010, accepted 16 April 2010

Published online 23 April 2010

Keywords thermal expansion, layered compounds

${ }^{*}$ Corresponding author: e-mail joseph.grima@um.edu.mt, Phone: +356 2340 2274, Fax: +356 25401091

A construct in the form of a multi-layered system built from conventional materials having different mechanical and thermal properties is proposed. This construct can exhibit predetermined values of thermal expansion which could also be negative. It is shown that for maximising negative thermal expansion (NTE) there should be a combination of thin layers of stiff materials having a high positive coefficient of thermal expansion (CTE) bonded with thicker layers of a soft material having low CTE values and, more importantly, having Poisson's ratio as high as possible.
The extent to which systems (materials or structures) change shape when subjected to changes in temperature is a subject of great practical importance and has been studied for many years. It is well known that miscalculations of the temperature effects in the design of objects which are subjected to significant temperature changes can lead to disastrous consequences. This led to various studies aimed at designing, analysing, manufacturing and/or testing of materials having very particular coefficients of thermal expansion [1-18] (including materials exhibiting negative coefficients of thermal expansion, i.e. materials which contract when heated [2-17]). Of particular interest are composites having pre-determined coefficients of thermal expansion which are already being used in some niche applications, although there is still an ongoing need to develop simple and non-expensive methods for achieving the same effect on any scale.

Here we propose and discuss a multi-layered system constructible at any length scale from conventional materials having different mechanical and thermal properties which may be combined to form systems which exhibit pre-determined values of thermal expansion which could also be negative. In particular we show that to optimise negative thermal expansion (NTE) characteristics, the systems must be constructed based on what is illustrated in
Fig. 1, i.e. by combining thin layers of stiff materials having a high positive coefficient of thermal expansion (CTE) characteristics bonded together through thicker layers of a soft material having low CTE values and, more importantly, having Poisson's ratio as high as possible, in analogy to work presented earlier [15-17].

To simplify our discussion we discuss first the optimal requirements for one of the most basic systems operating through the mechanism we are proposing which can be tailored to exhibit NTE, i.e. the system in Fig. 1(a). As illustrated in Figs. 1(a) and 2, this system may be described as a sandwich structure made from three layers of two different materials $\mathrm{A}$ and $\mathrm{B}$ having different thicknesses, thermal and mechanical properties which are perfectly bonded together.

Let us assume that this sandwich is in the form of a cuboid of overall dimensions $x \times y \times z$ at temperature $T$ where the $z$ dimension is given by $2 z_{\mathrm{A}}+z_{\mathrm{B}}, z_{\mathrm{A}}$ and $z_{\mathrm{B}}$ being the thicknesses of layers $\mathrm{A}$ and $\mathrm{B}$ at temperature $T$, respectively. Let us also assume that (i) materials A and B are isotropic; (ii) that material $\mathrm{A}$ is much stiffer than material B, i.e. $E_{\mathrm{A}} \gg E_{\mathrm{B}}$, where $E_{\mathrm{A}}$ and $E_{\mathrm{B}}$ are the Young's moduli of materials $\mathrm{A}$ and $\mathrm{B}$, respectively, and (iii) that necking effects may be ignored. Given these assumptions, if the system is subjected to a change in temperature $\Delta T$, 


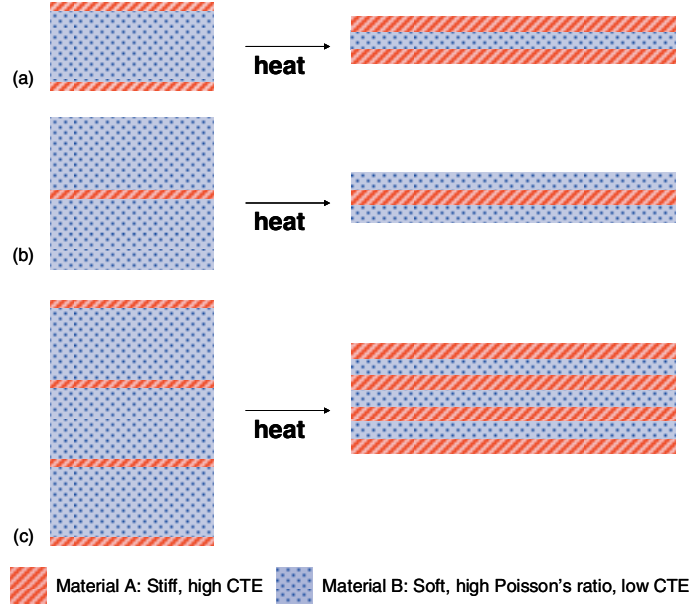

Figure 1 (online colour at: www.pss-rapid.com) An illustration of the concept proposed here illustrated for systems having three or more layers. Note that this system is highly anisotropic and the observed NTE is only exhibited in the direction orthogonal to the layers (linear NTE) and not overall (volumetric NTE) as is observed in $\mathrm{Y}_{2} \mathrm{Mo}_{3} \mathrm{O}_{12}$ or $\mathrm{ZrW}_{2} \mathrm{O}_{8}$.

then the $x$ and $y$ dimensions of the two layers must remain equal to each other even if materials $\mathrm{A}$ and $\mathrm{B}$ have different thermal expansion coefficients $\alpha_{\mathrm{A}}$ and $\alpha_{\mathrm{B}}$, respectively, since we are assuming that the layers are perfectly bonded together. In fact, given that we are assuming that $E_{\mathrm{A}} \gg E_{\mathrm{B}}$, the thermal expansion in the $x$ and $y$ dimensions is dictated primarily by the expansion of material $\mathrm{A}$ and may be approximated by $\Delta x=x \alpha_{\mathrm{A}} \Delta T$ and $\Delta y=y \alpha_{\mathrm{A}} \Delta T$. In contrast to this, the thickness in the third dimension will change by an amount

$$
\Delta z=2 \Delta z_{\mathrm{A}}+\Delta z_{\mathrm{B}},
$$

where $\Delta z_{\mathrm{A}}$ is the change in thickness of each of the A layers and is simply given by

$$
\Delta z_{\mathrm{A}}=z_{\mathrm{A}} \alpha_{\mathrm{A}} \Delta T,
$$

whilst $\Delta z_{\mathrm{B}}$, the change in thickness of the $\mathrm{B}$ layer, is caused by both the normal thermal expansion effect and also by deformations in material $\mathrm{B}$ so as to accommodate the mechanical constraint that the $x$ and $y$ dimensions remain the same as those of material A to which material B is perfectly bonded to. Thus, since material B is isotropic,

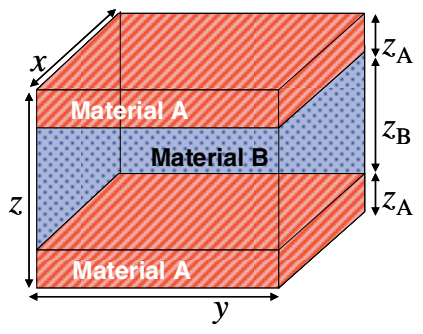

Figure 2 (online colour at: www.pss-rapid.com) Dimensions of the basic system discussed here.
$\Delta z_{\mathrm{B}}$ is given by [19]

$$
\Delta z_{\mathrm{B}}=z_{\mathrm{B}}\left[\alpha_{\mathrm{B}} \Delta T-\frac{2 v_{\mathrm{B}} \varepsilon_{\mathrm{AB}}}{1-v_{\mathrm{B}}}\right],
$$

where $v_{\mathrm{B}}$ is the Poisson's ratio of material B and $\varepsilon_{\mathrm{AB}}$ is the strain caused by material $\mathrm{A}$ on material $\mathrm{B}$ as a consequence of materials $\mathrm{A}$ and $\mathrm{B}$ having different thermal expansion coefficients which is given by

$$
\varepsilon_{\mathrm{AB}}=\left(\alpha_{\mathrm{A}}-\alpha_{\mathrm{B}}\right) \Delta T .
$$

By combining Eqs. (1)-(4), it becomes clear that

$$
\Delta z=\left[2 z_{\mathrm{A}} \alpha_{\mathrm{A}}+z_{\mathrm{B}} \alpha_{\mathrm{B}}-2 z_{\mathrm{B}} v_{\mathrm{B}} \frac{\alpha_{\mathrm{A}}-\alpha_{\mathrm{B}}}{1-v_{\mathrm{B}}}\right] \Delta T,
$$

which will be negative for a positive $\Delta T$ (heating) if

$$
2 z_{\mathrm{B}} \nu_{\mathrm{B}} \frac{\alpha_{\mathrm{A}}-\alpha_{\mathrm{B}}}{1-v_{\mathrm{B}}}>2 z_{\mathrm{A}} \alpha_{\mathrm{A}}+z_{\mathrm{B}} \alpha_{\mathrm{B}}
$$

For example, if our system is heated and material A expands more than material $\mathrm{B}$, then provided that $\nu_{\mathrm{B}}>0$, the 'mechanical' term $-2 z_{\mathrm{B}} v_{\mathrm{B}}\left(\alpha_{\mathrm{A}}-\alpha_{\mathrm{B}}\right)\left(1-v_{\mathrm{B}}\right)^{-1} \Delta T$ will have a negative magnitude, i.e. the in-plane expansion of the A layers causes a shrinkage in the out-of-plane $(z)$ dimension of the $\mathrm{B}$ layer. If this shrinkage is large enough to nullify the 'thermal' terms $\left[2 z_{\mathrm{A}} \alpha_{\mathrm{A}}+z_{\mathrm{B}} \alpha_{\mathrm{B}}\right] \Delta T$, there will be an overall contraction in thickness $(z)$ dimension when the system is heated, i.e. negative thermal expansion.

From these equations, it is clear that to magnify the NTE, in addition to having material $\mathrm{B}$ being much softer than material A, one should also have material B with as high a positive Poisson's ratio as possible (i.e. material B must be a rubber-type material). In fact, it is this Poisson's/rubber effect which is the essential feature for this mechanism to operate and generate NTE. Also, the CTE of material A must be as large as possible relative to that of material B so as to set the Poisson's effect in operation. However, so as to ensure that expansion in A caused by its high CTE does not nullify the contraction in $\mathrm{B}$ caused by the Poisson's effect, the A layers should be as thin as possible when compared to the B layer. In other words, as stated above, for maximum NTE, one should combine thin layers of stiff materials having high positive CTE characteristics bonded together through thicker layers of a soft material having low CTE values and more importantly, having Poisson's ratio as high as possible. Note that as illustrated in Fig. 1, similar properties will be obtained by systems having different numbers of layers, etc. (provided that care is taken so that their construction avoids bending caused by the well known bi-material strips effect).

To obtain further evidence that what is proposed here can indeed result in negative thermal expansion in the out-of-plane direction, we used the Finite Elements (FE) 


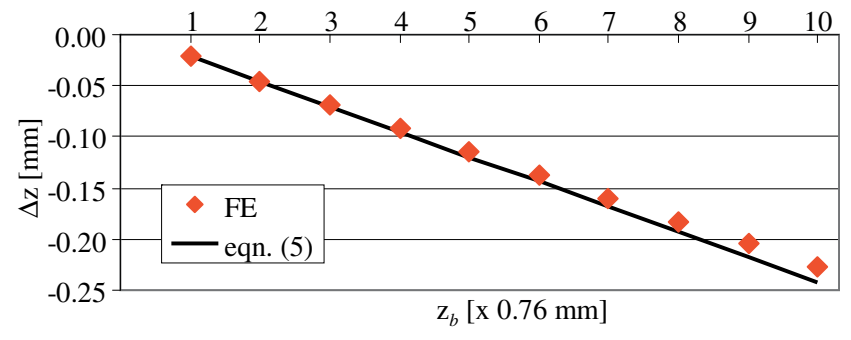

Figure 3 (online colour at: www.pss-rapid.com) Change in thickness as predicted by the analytical expression in Eq. (5) and by the FE simulations as a function of the thickness of layers of the soft material when the system is subjected to a change in temperature of $100 \mathrm{~K}$. Note that these systems all exhibit NTE the magnitude of which may be controlled by the thickness $z_{\mathrm{b}}$.

software package ANSYS Academic Research V.12.0 to construct a multilayered structure consisting of alternating layers of two elastic materials A and B which were perfectly bonded together and meshed using the 3-D, 20-node, coupled-field SOLID226 element. These simulations confirmed that it is indeed possible for this mechanism to operate. For example, when we performed simulations varying $z_{\mathrm{B}}$ having $x=y=200 \mathrm{~mm}, z_{\mathrm{A}}=0.076 \mathrm{~mm}$, $E_{\mathrm{A}}=0.552 \mathrm{GPa}, E_{\mathrm{B}}=0.000517 \mathrm{GPa}, \alpha_{\mathrm{A}}=1.9 \times 10^{-4} \mathrm{~K}^{-1}$ and $\alpha_{\mathrm{B}}=1.90 \times 10^{-5} \mathrm{~K}^{-1}, v_{\mathrm{A}}=0.333, v_{\mathrm{B}}=0.499$ we found that NTE would be exhibited, this being in close conformity with what was predicted by Eq. (5) (see Fig. 3). Note that material $\mathrm{A}$ has the properties of a typical polyvinylidene chloride and material $\mathrm{B}$ has the properties of a typical silicone rubber [20]. Here it should be emphasised that the simulations presented here are only meant to act as a 'proof of concept' and more detailed studies need to be carried out before choosing the materials (e.g. the material used in the simulations for the layer A has the limitation of being brittle, has low flex resistance and flakes off easily). One should also be careful to use appropriate ratios for $x / z$ and $y / z$ to ensure mechanical stability as otherwise, one may deviate from the requirement that the geometry of the layers is flat.

Before we conclude it is important to highlight that the mechanism proposed here is not only of use to generate NTE but also as a mechanism of producing systems with pre-desired mechanical properties. In fact, it should be emphasised that the exact magnitude of the thermal expansion coefficient in the thickness dimension may be adjusted not only through careful choice of materials A and B, but also through control of the relative thickness of the layers. It should also be noted that what is proposed here is a scale independent mechanism, i.e., it could theoretically be possible to implement this effect at any scale of structure provided the right components can be made available, as is normally the case with models for negative Poisson's ratio [8] or negative compressibility [21].

We hope that given the simplicity of the proposed system and its adjustability, the proposed construct or varia- tions of it will be manufactured and find use in practical applications.

Acknowledgements The support by the Malta Council of Science and Technology through their 2006 programme is gratefully acknowledged as is the support of Ing. Michael Attard of IMA Engineering Malta and of Professor Krzysztof W. Wojciechowski of the Polish Academy of Sciences.

\section{References}

[1] R. E. Taylor, in: Thermal Expansion of Solids, edited by C. Y. Ho (ASM International, 1998).

[2] J. S. O. Evans, J. Chem. Soc., Dalton Trans., 3317 (1999).

[3] A. W. Sleight, in: Materials Research Society Symposium Proceedings, Vol. 755, edited by M. Greenblatt, M. A. Alario-Franco, M. S. Whittingham, and G. Rohrer (MRS, 2002), DD10.6.

[4] G. D. Barrera, J. A. O. Bruno, T. H. K. Barronand, and N. L, Allan, J. Phys.: Condens. Matter 17, R217 (2005).

[5] J. N. Grima and K. W. Wojciechowski, Phys. Status Solidi B 245(11), 2369 (2008), Preface to the Special Issue and papers cited therein.

[6] L. J. Vandeperre and W. J. Clegg, Tailoring Strains through Microstructural Design, in: Materials Research Society Symposium Proceedings, Vol. 785, edited by Y. Furuya, E. Quandt, Qiming Zhang, K. Inoue, and M. Shahinpoor (MRS, 2003), D11.4.

[7] C. W. Smith, W. Miller, D. S. Mackenzie, and K. E. Evans, Mechanism for Negative Thermal Expansion and Its Links to Negative Poisson's Ratio, in: Second International Workshop on Auxetic and Related Systems, Poznan, Poland, 2005.

[8] J. N. Grima, P. S. Farrugia, R. Gatt, and V. Zammit, Proc. Roy. Soc. A 463, 1585 (2007).

[9] T. Mary, J. S. O. Evans, T. Vogt, and A. W. Sleight, Science 272, 90 (1996).

[10] R. S. Lakes, J. Mater. Sci. Lett. 15, 475 (1996).

[11] O. Sigmund and S. Torquato, Appl. Phys. Lett. 69, 3203 (1996).

[12] O. Sigmund and S. Torquato, J. Mech. Phys. Solids 45, 1037 (1997)

[13] G. Milton, The Theory of Composites (Cambridge University Press, 2002).

[14] W. Miller, D. S. Mackenzie, C. W. Smith, and K. E. Evans, Mech. Mater. 40, 4/5 (2008).

[15] A. Kelly, R. J. Stearn, and L. N. McCartney, Compos. Sci. Technol. 66, 2 (2006).

[16] A. Kelly, L. N. McCartney, W. J. Clegg, and R. J. Stearn, Compos. Sci. Technol. 65, 1 (2005).

[17] M. Landert, A. Kelly, R. J. Stearn, and P. J. Hine, J. Mater. Sci. 39, 11 (2004).

[18] A. Alderson, K. L. Alderson, G. D. Hudson, and D. E. Skertchly, European Patent EP1989249 B1 (2009).

[19] D. W. A. Rees, The Mechanics of Solids and Structures (McGraw-Hill, UK, 1990).

[20] http://www.efunda.com/materials/polymers (accessed $30 \mathrm{Ju}-$ ly 2009).

[21] R. Gatt and J. N. Grima, Phys. Status Solidi RRL 2, 236238 (2008). 